\title{
A bowed serial-position function in habituation of sequential stimuli
}

\author{
ALLAN R. WAGNER and PENN L. PFAUTZ \\ Yale University, New Haven, Connecticut 06520
}

\begin{abstract}
The effect of serial position was examined in a study of habituation of the vasoconstrictive orienting response in rabbits. In a procedure analogous to a recognition memory test, subjects received multiple exposures to an invariant list of stimuli before their response was assessed to an isolated item that had consistently occupied either the first, middle, or last position in the list. A reliably bowed serial-position function was obtained, with the least habituation (poorest retention) occurring for the item that had occupied the middle position in the list. The finding of both a primacy and a recency effect parallels the most general finding in studies of human memory and encourages a compatible account of habituation in infrahuman subjects. The result was discussed in terms of the opportunities for differential rehearsal of list items and contextual cues at different positions in the list.
\end{abstract}

In approaching most learning phenomena, the theorist is likely to be especially sensitive to the relationships that obtain among the separable stimuli in the training environment and to be attentive to how these relationships may have contributed to the phenomena. The notable exception to this attitude is the case of habituation. Research and theorizing have largely proceeded in keeping with a general bias, that habituation follows only from the organism's experience with the stimulus in question and is indifferent to the characteristics of the surrounding stimulation. The common definition of habituation, as a "response decrement resulting from repeated stimulation," is meant, for example, to exclude behavioral products attributable to the stimulus relationships of associative learning (see Hinde, 1970; Thorpe, 1956). But, research on habituation has rarely addressed this particular exclusion. Given this attitude, it is not surprising that Thompson and Spencer (1966), in listing the parametric characteristics of habituation in their authoritative review, make no mention of any known influences of contemporaneous stimulation on the development of habituation.

In a departure from this tradition, Wagner (1976, 1978) has proposed a theory of habituation in which contemporaneous stimulation is seen as no less important than in any learning situation. Basically, the theory involves an information-processing approach in which the consequences of stimulus exposure are assumed to be modulated by the limited

The research reported was supported in part by National Science Foundation Grant BMS74-20521 to Allan R. Wagner. Portions of this paper were presented at the meeting of the Psychonomic Society, Washington, D.C., November 1977. Requests for reprints should be addressed to Allan R. Wagner, Department of Psychology, Yale University, New Haven, Connecticut 06520. capacity of an active, short-term memory (STM) process and to be importantly determined by the opportunities for associative learning, which requires representational contiguity in STM. The details of the theory are relatively unimportant to the present purposes. The essential notion, however, is that a response decrement attributable to "habituation" results from the stimulus's representation being preactivated in STM on the occasion of testing. This presumably may occur as a consequence of a perseverating representation from recent exposure to the same stimulus or as a consequence of a recent activation of a corresponding representation in the long-term memory (LTM) network via associated contextual stimuli. The former influence is taken to be responsible for a short-term refractory-like habituation effect (e.g., Davis, 1970; Whitlow, 1975) and the latter for a more permanent habituation effect (e.g., Davis, 1970; Wagner, 1976) detectable on remote retention tests.

Some specific predictions of this formulation, concerning the influences of contemporaneous stimulation on habituation, have been supported. Thus, there is some evidence that the relatively permanent habituation effect requires the same stimulus context as was present during habituation (e.g., Peeke \& Veno, 1973) and can be "extinguished" by exposing the subject to the context in the absence of the habituation stimulus (e.g., Wagner, 1976). There is also some evidence (Wagner, 1976) that the development of a persistent response decrement can be interfered with by the poststimulation presentation of a "distractor" that can otherwise be assumed to compete for active status in STM (Whitlow, 1975) and thus interfere with the formation of associations.

The present investigation was concerned with a 
more empirical possibility. If a subject is repetitively exposed to a target stimulus, but always within a list of other stimuli, will the sensitivity of habituation to contemporaneous stimulation be evidenced in a serial position function when the target is presented in isolation on a remote retention test? One of the most ubiquitous findings in the literature on learning and memory is the bowed serial-position curve (see Crowder, 1976, for a review), with items experienced in the middle of the list being less well remembered than those at either the beginning of the list (which are said to show a "primacy effect") or at the end of the list (which are said to show a "recency effect"). It is in the general sense of Wagner's (1976) view of habituation to anticipate that this empirical phenomenon would be no less characteristic of habituation.

The possibility was evaluated using the evoked vasomotor response in the rabbit, as has been used in more specific tests of Wagner's treatment of habituation (e.g., Wagner, 1976, 1978; Whitlow, 1975). The employment of infrahuman subjects makes the evaluation the more interesting as (a) theoretical treatments of the bowed serial-position function are often dependent upon assumptions peculiar to the human (e.g., Feigenbaum \& Simon, 1962) and (b) there is no substantial literature on serial position effects with infrahuman subjects, except in immediate memory tasks that favor a recency effect on a simple temporal basis (e.g., Shimp \& Moffitt, 1974; Thompson \& Herman, 1977; Whitlow, 1975).

\section{METHOD}

\section{Subjects}

Subjects were 48 male albino New Zealand rabbits obtained from a local breeder and weighing $2-3 \mathrm{~kg}$. They were housed in individual cages with free access to food and water.

\section{Apparatus}

During experimental sessions, the subjects were loosely restrained in a $51 \times 18 \times 14 \mathrm{~cm}$ Plexiglas box through which only the head protruded. The restraining box was placed inside a $107 \times 84 \times 79 \mathrm{~cm}$ isolation chamber illuminated by a red $100-\mathrm{W}, 115-\mathrm{V}$ bulb supplied with $60 \mathrm{~V}$ by a variable transformer. White noise delivered through a speaker located above and to the left rear of the subject provided an ambient sound level of $72-74 \mathrm{~dB}\left(\mathrm{re} 20 \mu \mathrm{N} / \mathrm{m}^{2}\right)$ as measured at the locus of the subject's head (General Radio sound pressure level meter, Model 1551). Temperature inside the chamber was maintained at $25^{\circ}-27^{\circ} \mathrm{C}$.

Five stimuli were employed in the experiment. A $530-\mathrm{Hz}$ tone generated by a Hewlett-Packard Model 200ABR audio oscillator could be delivered through the same speaker as the white noise and raised the sound pressure level to $82-84 \mathrm{~dB}$. A second auditory stimulus was provided by a $30 / \mathrm{sec}$ train of clicks created by a relay in a metal sounding box located on the floor of the chamber to the right rear of the subject, raising the sound level to $82 \mathrm{~dB}$. A visual stimulus was provided by two unsynchronized flashing lights (Grass Photostimulators, Model PS-2, set for 3.5 flashes $/ \mathrm{sec}$ and a nominal intensity of 16 ) mounted in front of and $40 \mathrm{~cm}$ above the subject. During presentation of this stimulus, the houselight was extinguished. A $60-\mathrm{Hz}$ vibrotactile stimulus was provided by a Valmour Model 880 hand massager mounted on the floor of the restraining box and in firm contact with the subject's chest. Finally, an Argonaut Model LRA 046 constant current stimulator provided an electrotactile stimulus consisting of a $5-\mathrm{Hz}$ train of $2-\mathrm{mA}, 0.5-\mathrm{msec}$ square waves. This stimulus was delivered through two stainless steel electrodes (Sklar surgical wire, 32-ga) implanted in the skin about the orbit of the subject's left eye. One electrode was approximately $5 \mathrm{~mm}$ below the extreme nasal extent of the eye and the other was approximately $5 \mathrm{~mm}$ above the extreme lateral extent. This stimulus was developed, with the parameters described, for use with human subjects to mimic discrete tactile stimulation (Geldard \& Sherrick, 1972), and produced in the rabbit, as prepared here, a faint twitching of the eyelid without apparent aversiveness. All stimuli were $1 \mathrm{sec}$ in duration.

Vasomotor activity was recorded with a photoelectric plethysmograph affixed to the subject's right ear in a region $5 \mathrm{~cm}$ from the base and $3 \mathrm{~cm}$ from the tip of the ear, between the auricular artery and the posterior auricular vein. The transducer consisted of a Chicago Miniature light bulb (CM713), powered by a $4-\mathrm{V}$ supply, and a Clairex CL903A photocell aligned such that the light bulb faced the photocell across the ear tissue. Changes in blood volume altered the amount of light transmitted through the ear tissue, thereby altering the conductance of the photocell, which served as one arm of a $6.25-\mathrm{V}$ bridge circuit. Voltage changes associated with changes in conductance were graphically recorded on a Beckman Dynograph after passing through a dc coupling and a low-pass filter that attenuated, by at least $3 \mathrm{~dB}$, frequencies greater than $.5 \mathrm{~Hz}$. The filtered signal was also digitized by a PDP $11 / 10$ computer system and stored for later analysis.

\section{Procedure}

Two days prior to testing, the subjects were introduced to the conditions of confinement by being placed in the restraining box for approximately $2 \mathrm{~h}$. During this time, one ear was shaved and depilated and the paraorbital electrodes were implanted. On the next day, the subjects were placed in the restrainer inside the experimental chamber with the plethysmograph in place for approximately $1 \mathrm{~h}$.

The experimental manipulation took place on the day following adaptation to the chamber. The session began with $15-20 \mathrm{~min}$ of adaptation to the apparatus, at the end of which time the acceptability of the preparation was assessed by introducing a .2-V decrease in the 4-V power supply to the transducer light. If the conductance changed by $25 \mu \mathrm{S}$ or less, the preparation was considered acceptable. If the conductance was greater than $25 \mu \mathrm{S}$, the transducer was adjusted to encompass more ear tissue. In addition to this calibration check, the responsivity of the animal to opening the door of the isolation chamber also provided an indication of the adequacy of transducer placement. Lack of a recognizable vasoconstriction response to door opening resulted in alteration of the placement. When the preparation was judged acceptable according to these criteria, presentation of experimentally programmed stimuli began 5 min later.

For all subjects, the electrotactile stimulus was the event to which habituation was assessed. The stimulus was presented on 8 or 16 occasions (see below), each time being one item in a 5- or 9-item list (see below) constructed with the remaining stimuli previously described. After a retention interval, the stimulus was then presented alone to evaluate the degree of vasomotor responding that was evoked. The experimental manipulation of interest was that for 16 subjects the to-be-tested electrotactile stimulus was always the first item in the list presentations, for 16 it was always the middle item, and for the remaining 16 it was always the last item.

During the list habituation phase, the interstimulus intervals within the list were uniformly $2 \mathrm{sec}$ (onset to onset) and the interlist intervals were $150 \mathrm{sec}$. For any subject, the sequence of stimuli within the list was invariant on the several list presentations, 
and involved either a single occurrence of each of the other four stimuli in addition to the electrotactile stimulus (for five-item lists) or two nonadjacent occurrences oi each of the other four stimuli (for nine-item lists).

In order to preclude the possibility that any serial position effects could be peculiar to a particular local relationship between the electrotactile stimulus (E) and one of the remaining light $(L)$, vibratory $(V)$, tonal $(T)$, or click (C) stimuli, different subjects in each major condition were run with different orders of the remaining stimuli, with the following constraint. For each subject run with the electrotactile stimulus in the middle position (e.g., with the list VTECL), a corresponding subject was run with the electrotactile stimulus in the initial position, immediately followed by the same items (e.g., with the list ECLTV), and another corresponding subject was run with the electrotactile stimulus in the last position, immediately preceded by the same items (e.g., with the list LCVTE).

The number of items in the list and the number of list presentations was varied across four replications, each of which included four subjects from each of the three serial-position groups, matched as indicated above for list structure. In two of the replications, the lists contained five items, while for the remaining two the lists contained nine items. One of each of these pairs of replications employed eight list repetitions, while the other employed 16 list repetitions.

For all subjects, the test phase began $15 \mathrm{~min}$ after the last list presentation. During this retention interval, no stimuli were presented. The test series consisted of 12 presentations of the 1 -sec electrotactile stimulus by itself, at interstimulus intervals of $150 \mathrm{sec}$.

\section{Scoring}

Quantitative analysis of vasomotor responding over the final test series was based on averaged waveforms $f \cap r$ individual subjects. The output voltage of the plethysmograph was sampled every $.5 \mathrm{sec}$ from $5 \mathrm{sec}$ before to $20 \mathrm{sec}$ after each stimulus onset. A baseline value was defined on each trial as the mean of the 11 samples prior to and including the moment of stimulus onset, and all trial values were expressed as voltage deviations from baseline. The deviation values at each sampling interval were then averaged across trials for each subject. Similar procedures were employed to inspect vasomotor responding over the $5 \mathrm{sec}$ before and the $20 \mathrm{sec}$ after the initiation of the list of stimuli during habituation.

\section{RESULTS}

The sluggishness of the vasomotor response precluded independent assessment of the vasoconstriction provoked by the electrotactile stimulus of interest during the list habituation phase, i.e., separate from that provoked by the remaining items on the list. Figure 1 presents the averaged vasoconstriction responses to the first two and last two list presentations for all subjects receiving five-item lists (left panel) and all subjects receiving nine-item lists (right panel). It is apparent that the separate waveforms do not reveal any discontinuities timelocked to the separate stimuli following the first item. The only discernible effect that can be attributed to the differential withinlist stimulation can be seen in comparing the waveforms to the five- and nine-item lists beyond the point at which they differ, i.e., after the presentation of the sixth item in the latter list: Over the last $8 \mathrm{sec}$ of the 20 -sec sampling period, the averaged response to the five-item list was returning to baseline

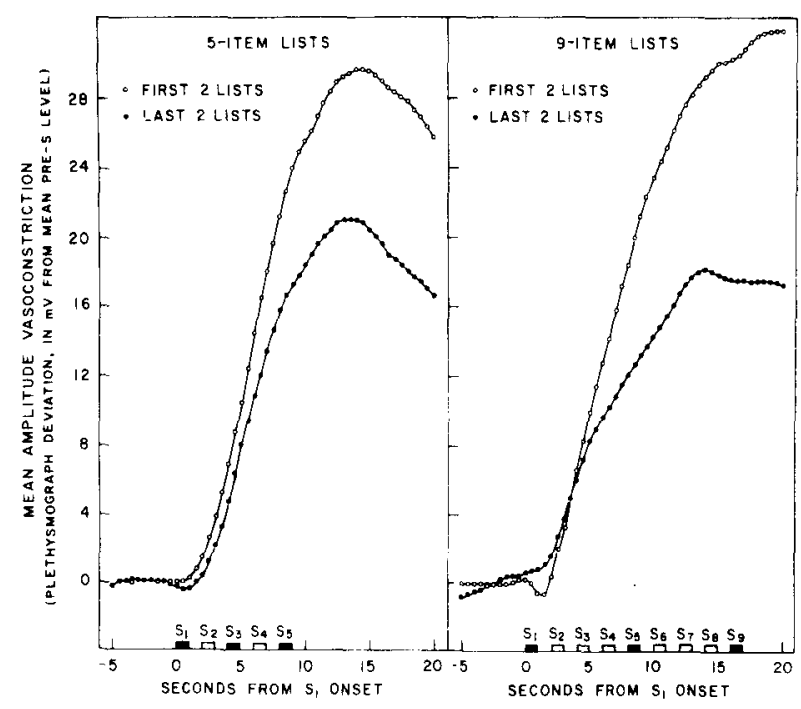

Figure 1. Mean average evoked response to the first and last two list presentations for those subjects receiving a five-item list (left panel) and those receiving a nine-item list (right panel) containing a to-be-tested electrotactile stimulus. The item occasions are indicated on the abscissa, with the three possible locations of the electrotactile stimulus (at either the start, middle, or end of the list, for different subjects) marked by solid bars.

while such return was not yet as apparent in the averaged response to the nine-item list.

Habituation of the vasoconstriction response to the stimulus list is apparent in Figure 1, in the reduced amplitude of response on the final two presentations as compared to the first two presentations. To summarize this change, the mean deviation from baseline was computed over the entire 20 -sec period following the initial list stimulus at each of the two stages of training. According to this measure, there was a statistically significant response decrement $[\mathrm{F}(1,44)=26.58, \mathrm{p}<.01]$ that was not reliably different $[F(1,44)=.28, p>.25]$ in the case of the two list lengths. It may also be noted that the subgroups that received 16 list presentations did not show reliably more response decrement by the end of the habituation series than the subgroups that received 8 list presentations $[F(1,44)=.57, p>.25]$. Consequently, this variable has been ignored in the display of Figure 1.

The data of principal interest are from the retention test responses to the electrotactile stimulus alone, in the three major groups for which that stimulus had occupied the first, middle, or last position in the habituation lists. Figure 2 presents the mean evoked responses, averaged over the 12 test presentations, for each of the three groups of 16 subjects. It may be seen that the magnitude of the response to the isolated stimulus over the trials included is appreciably less than that seen earlier to the list of stimuli, as depicted in Figure 1. The $20-\mathrm{sec}$ poststimulus sampling period here includes 


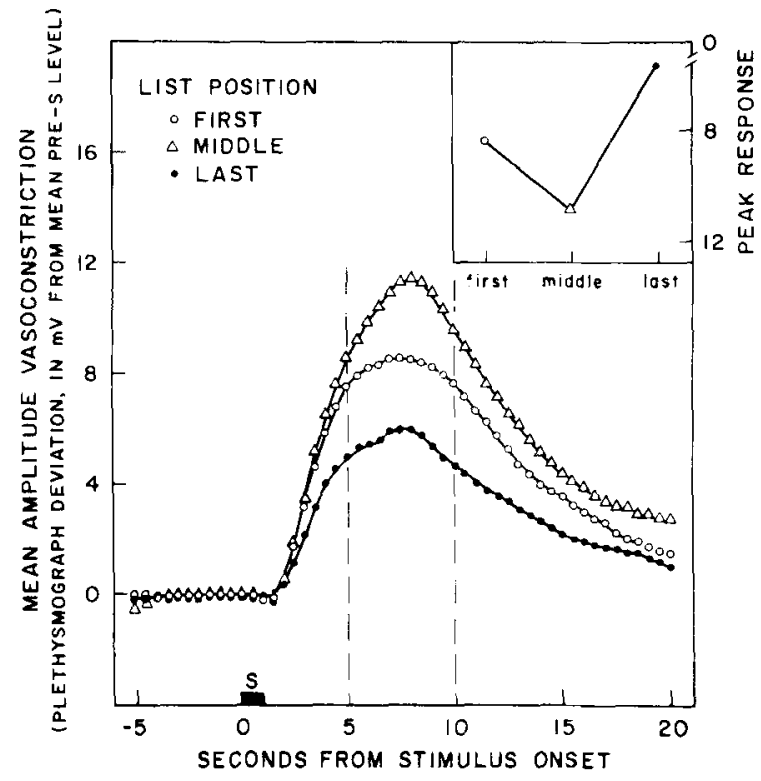

Figure 2. Mean average evoked response to the electrotactile stimulus alone, for each of the three groups distinguished by whether the stimulus had been experienced in the first, middle, or last position in the habituation list. The insert presents the differential peak responses (average of the points between 5 and 10 sec poststimulus, included within the broken lines of the main figure) as a serial position function. The ordinate scale is inverted in this case to suggest the better retention that is indicated by the smaller evoked response.

essentially the full course of the response, which for all groups peaked within the interval from 5 to $10 \mathrm{sec}$ after stimulation.

The notable finding is obvious in the full waveforms of the three groups of Figure 2. There was the greatest amplitude of response in the group for which the stimulus occupied the middle position in the lists, with lesser amplitudes of response in the groups for which the stimulus occupied the first or last positions in the list, in that order. An alternative summary of this finding is presented in the insert to Figure 2. The average response amplitude over the 5- to 10-sec poststimulus period, which embraced the peak activity, is plotted as a function of the serial position the stimulus occupied in the three groups. In this case, the abscissa values have been inverted in keeping with the view that the greater the responsiveness to the test stimulus, the less retention of habituation was detectable. Clearly, retention was a nonmonotonic function of serial position, with the least retention (greatest vasoconstriction) for a stimulus in the middle as compared to the first or last positions in the list.

Statistical analyses were conducted on the averaged responses over the 5- to 10 -sec poststimulus interval as depicted in Figure 2. In these analyses, subjects from the three groups were arrayed in matched triplets according to the manner in which they had been assigned corresponding lists (see Procedure), while list length and number of list presentations were included as orthogonal betweentriplet variables. There was a reliable overall difference among the three serial position groups $[F(2,24)$ $=4.07, \mathrm{p}<.05]$. Confirmation of the nonmonotonic nature of the serial position effect was provided by the finding of a significant quadratic trend $[F(1,24)$ $=5.69, \mathrm{p}<.05]$ and $\mathrm{a}$ nonsignificant residual $[F(1,24)=2.24, p>.10]$. Of the 16 triplets in the study, 11 , or $69 \%$, showed more vasoconstriction (less apparent habituation) to the stimulus when it had been experienced in the middle of the list than when experienced at either the beginning or the end of the list. This percentage in comparison to the chance value of $33 \%$ is further indication of the reliability $(\mathrm{z}=2.74, \mathrm{p}=.003)$ of the bowed serialposition function as averaged in Figure 2.

In advance of the present study, it could not be known what conditions of list length and number of list presentations would allow the detection of any serial position effects that might obtain. Hence, variations in each were included across the several replications. In fact, neither of the variables, at the values compared, had any discernible influence on the overall level of responding during testing or was involved in any reliable interactions with the serial position effect.

\section{DISCUSSION}

The habituation observed to an isolable stimulus may be no less dependent upon the context of events in which the stimulus is experienced than are other measures of learning and memory. The present study points in this direction by suggesting that habituation of the vasomotor reaction in the rabbit is not immune to the primacy and recency effects that combine to yield a bowed serial-position function. In conjunction with other recent findings (e.g., Wagner, 1976), it thus encourages a theoretical approach to habituation that would entertain the same episodic processing interactions as are characteristically acknowledged in systematic interpretations of other instances of learning (e.g., Atkinson \& Shiffrin, 1968; Wagner, 1978).

It is instructive in this regard to compare the present findings with those of Whitlow (1975), who used the same habituation preparation and similar five-item lists of stimuli, but with the intention of monitoring the transient decrement in response that could be attributed to the location of the test item in an immediately preceding list, as opposed to the cumulative history of prior exposures. To this end, Whitlow (1975) varied the location of an individual item within the lists from occasion to occasion, so as to insure that the to-be-tested stimulus was 
equally experienced in each position. Test presentations of the item in isolation were then arranged $40 \mathrm{sec}$ after different lists in which it had occupied the first, middle, or last position. Under such circumstances, Whitlow (1975) observed a monotonic recency effect, i.e., less vasoconstriction (more evidence of habituation), the closer to the end of the list (and to the test stimulus) that the item had occurred on that trial. Wagner (1976) and Whitlow (1975) attributed this effect ot the differential likelihood that representation of the to-be-tested item was allowed to remain active in STM from the time of its list occurrence until the time of testing, in the face of competition for occupancy of STM from the different numbers of intervening items in the remainder of the list.

In contrast to the Whitlow (1975) study, the design of the present investigation was calculated to minimize the liklihood that test responding would be singularly influenced by the transient state of STM immediately following list presentations and to increase the chances of detecting differences in the more durable overall products of stimulus exposure in the different serial positions. This was done by employing a delayed retention test following multiple presentations of a consistently ordered list. And in this case there was evidence of a primacy effect as well as a recency effect.

The differential outcome of these two studies is just what one would have expected from related investigations of human memory. When conditions are arranged to be especially sensitive to what stimulus representations may perseverate in STM shortly following list exposure, there is a pronounced recency effect (e.g., Murdock, 1962). When these conditions are removed by using a delayed test (e.g., Craik, 1970) or by interspersing distractor tasks between list presentations and testing (e.g., Glanzer \& Cunitz, 1966), the recency effect is diminished, to make the primacy effect more prominent. Indeed, Craik (1970) and Rundus, Loftus, and Atkinson (1970) have reported a monotonic primacy effect with delayed testing. Although it is clear from other human data (see Crowder, 1976), in agreement with the present study, that conditions that presumably allow persistence of item representation in STM until the occasion of testing are not necessary for the recency effect, they do favor such an effect in comparison with a primacy effect.

There are various interpretations of a bowed serial-position function on a remote retention test that might be applicable to the present findings. The interpretation of Feigenbaum and Simon (1962) has already been singled out as one that would not appear to be, in that it assumes a directing mnemonic strategy that is unlikely to be employed by rabbits without inducement to prepare for an eventual test.
Theories, such as that of Murdock (1960), that assume that the first and last items are more distinctive in training than are the middle items because of their unique locations cannot be dismissed, but do beg further sentences that specify the reasons that some locations are considered more unique than others (e.g., Bower, 1971). It is reasonable to assume that, in some fashion, the initial and terminal items in a list, as compared with the intermediate items, enjoy preferential occupancy of STM and thus are better learned about. It could be noted that the first items do not have to compete with preceding items for access to STM (e.g., Atkinson \& Shiffrin, 1968 ), and that the last items are not subject to displacement by following items (e.g., Norman \& Waugh, 1968). Again, however, such reasoning begs more precise characterization of the operating characteristics of STM, to see why the middle items are not at a net advantage, with fewer preceding items than the terminal items and fewer following items than the initial items.

Our tentative interpretation of the uniqueness and processing advantage of the initial and terminal items in a list, as compared to the more embedded items, is in their relationship to the contextual stimuli in the presence of which the items are eventually tested. That is, the contextual stimuli that are present at the time of testing are similar to those that are present prior to list presentation (i.e., adjacent to the initial item), and are again present following list presentation (i.e., adjacent to the terminal item), but are distinctly different from those that are present during the list itself (i.e., adjacent to the middle items). We can assume that these juxtapositions of items and context will favor the joint rehearsal in STM of either the initial or terminal items (as compared to middle items) along with the testappropriate context. Now, if the relatively permanent habituation effect observed to an item is context specific, as other measures of recognition have been supposed to be (e.g., Anderson \& Bower, 1972), we could be led to anticipate a bowed serial position function.

This reasoning is clearly speculative at this point. But it is congenial to Wagner's (1976) treatment of long-term habituation, which assumes that contextual stimuli associated with the test stimulus must prime a representation of that cue in STM to accomplish the reduction in responding that is observed.

\section{REFERENCES}

Anderson, J. R., \& Bower, G. H. Recognition and retrieval processes in free recall. Psychological Review, 1972, 79, 97-123. Atrinson, R. C., \& Shiffrun, R. M. Human memory: A proposed system and its control processes. In K. W. Spence \& J. T. Spence (Eds.). The psychology of learning and motivation (Vol. 2). New York: Academic Press, 1968. 
BOWER, G. H. Adaptation-level coding of stimuli and serial position effects. In M. H. Appley (Ed.), Adaptation-level theory. New York: Academic Press, 1971.

CraIK, F. I. M. The fate of primary memory items in free recall. Journal of Verbal Learning and Verbal Behavior, 1970, 9, 143-148.

Crowder, R. G. Principles of learning and memory. Hillsdale, N.J: Erlbaum, 1976.

DAvis, M. Effects of interstimulus interval length and variability on startle-response habituation in the rat. Journal of Comparative and Physiological Psychology, 1970, 72, 177-192.

Feigenbaum, E. A., \& Simon, H. A. A theory of the serial position effect. British Journal of Psychology, 1962, 53, 307-320.

Geldard, F. A., \& Sherrick, C. E. The cutaneous "rabbit": A perceptual illusion. Science, 1972, 178, 178-179.

Glanzer, M., \& Cunitz, A. R. Two storage mechanisms in free recall. Journal of Verbal Learning and Verbal Behavior, $1966,5,351-360$.

Hinde, R. A. Behavioural habituation. In G. Horn \& R. Hinde (Eds.), Short-term changes in neural activity and behavior. Cambridge: Cambridge University Press, 1970.

Murdock, B. B., JR. The distinctiveness of stimuli. Psychological Review, 1960, 67, 16-31.

Murdock, B. B., JR. The serial position effect of free recall. Journal of Experimental Psychology, 1962, 64, 482-488.

Norman, D. A., \& WaUgh, N. C. Stimulus and response interference in recognition memory experiments. Journal of Experimental Psychology, 1968, 78, 551-559.

Peeke, H. V. S., \& Veno, G. Stimulus specificity of habituated aggression in three-spined sticklebacks (Gasterosteus aculeatus). Behavioral Biology, 1973, 8, 427-432.
Rundus, D., Loftus, G. R., \& Atkinson, R. C. Immediate free recall and three-week delayed recognition. Journal of Verbal Learning and Verbal Behavior, 1970, 9, 684-688.

Shimp, C. P., \& Moffrt, M. Short-term memory in the pigeon: Stimulus-response associations. Joumal of the Experimental Analysis of Behavior, 1974, 22, 507-512.

Thompson, R. F., \& Spencer, W. A. Habituation: A model phenomenon for the study of the neuronal substrates of behavior. Psychological Review, 1966, 173, 16-43.

Thompson, R. K., \& Herman, L. M. Memory for lists of sounds by the bottle-nosed dolphin: Convergence of memory processes with humans? Science, 1977, 195, 501-503.

THORPE, W. H. Learning and instinct in animals. London: Methuen, 1956.

WAGNER, A. R. Priming in STM: An information-processing mechanism for self-generated or retrieval-generated depression in performance. In T. J. Tighe \& R. N. Leaton (Eds.), Habituation: Perspectives from child development, animal behavior, and neurophysiology. Hillsdale, N.J: Erlbaum, 1976.

WAGNER, A. R. Expectancies and the priming of STM. In S. H. Hulse, H. Fowler, \& W. K. Honig (Eds.), Cognitive processes in animal behavior. Hillsdale, N.J: Erlbaum, 1978.

Whrtow, J. W. Short-term memory in habituation and dishabituation. Joumal of Experimental Psychology: Animal Behavior Processes, 1975, 1, 189-206.

(Received for publication November 15, 1977; revision accepted April 28, 1978.) 